\title{
Abciximab (ReoPro) Dosing Strategy for the Management of Acute Intraprocedural Thromboembolic Complications during Pipeline Flow Diversion Treatment of Intracranial Aneurysms
}

\author{
Li-Mei Lin $^{a} \quad$ Bowen Jiang $^{b}$ Jessica K. Campos ${ }^{b}$ \\ Narlin B. Beaty ${ }^{b}$ Matthew T. Bender ${ }^{b}$ Rafael J. Tamargo ${ }^{b}$ \\ Judy Huang ${ }^{\mathrm{b}}$ Geoffrey P. Colby ${ }^{\mathrm{c}}$ Alexander L. Coon ${ }^{\mathrm{b}}$ \\ aDepartment of Neurosurgery, University of California, Irvine School of Medicine, \\ UC Irvine Medical Center, Orange, CA, USA; ${ }^{b}$ Department of Neurosurgery, Johns Hopkins \\ University School of Medicine, The Johns Hopkins Hospital, Baltimore, MD, USA; \\ 'Department of Neurosurgery, University of California, Los Angeles, Los Angeles, CA, USA
}

\section{Keywords}

Abciximab · Aneurysm · Endovascular treatment · Flow diversion · Pipeline embolization device $\cdot$ Thrombosis

\begin{abstract}
Background: Flow diversion with the Pipeline embolization device (PED) is an effective neuroendovascular method and increasingly accepted for the treatment of cerebral aneurysms. Acute in situ thrombosis is a known complication of PED procedures. There is limited experience in the flow diversion literature on the use of abciximab (ReoPro) for the management of acute thrombus formation in PED cases. Methods: Data were collected retrospectively on patients who received intra-arterial (IA) ReoPro with or without subsequent intravenous (IV) infusion during PED flow diversion treatment of intracranial aneurysms. Results: A total of 30 cases in patients with a mean age of 56.7 years (range 36-84) and a mean aneurysm size of $8.6 \mathrm{~mm}$ (range 2-25) were identified to have intraprocedural thromboembolic complications during PED treatment. IA ReoPro was administered in all cases, with 20 cases receiving increments of $5-\mathrm{mg}$ boluses and 10 cases receiving a $0.125 \mathrm{mg} / \mathrm{kg}$ IA bolus (half cardiac dosing). Complete or partial recanalization was achieved in $100 \%$ of the cases. IV ReoPro infusion at $0.125 \mu \mathrm{g} / \mathrm{kg} / \mathrm{min}$ for $12 \mathrm{~h}$ was administered postprocedurally in 22 cases with a residual throm-
\end{abstract}


bus. Postprocedurally, 18 patients were transitioned from clopidogrel (Plavix) to prasugrel (Effient). The majority of the cases $(23 / 30 ; 77 \%)$ were discharged home. Periprocedural intracranial hemorrhage was noted in 2 cases (7\%) and radiographic infarct was noted in 4 cases (13\%), with an overall mortality of $0 \%$ at the time of initial discharge. Clinical follow-up was available for $28 / 30$ patients. The average duration of follow-up was 11.7 months, at which time 23/28 (82\%) of the patients had a modified Rankin Scale score of 0. Conclusions: IA ReoPro administration is an effective and safe rescue strategy for the management of acute intraprocedural thromboembolic complications during PED treatment. Using a dosing strategy of either 5 - $\mathrm{mg}$ increments or a $0.125 \mathrm{mg} / \mathrm{kg}$ IA bolus (half cardiac dosing) can provide high rates of recanalization with low rates of hemorrhagic complications and long-term morbidity.

(c) 2018 S. Karger AG, Basel

\section{Introduction}

The Pipeline embolization device (PED; Medtronic Neurovascular, Irvine, CA, USA) and the second-generation PED Flex (Medtronic Neurovascular) have become increasingly pertinent modalities in the modern management of cerebral aneurysms [1-3]. The safety and efficacy of the PED have been demonstrated in both immediate and long-term outcome studies [2, 4-7]. Dual antiplatelet therapy is routinely used to mitigate known thromboembolic risks. The reported overall ischemic stroke risk for patients undergoing PED treatment ranges from 3 to $6 \%$ while on maintenance with dual antiplatelet therapy $[1,8,9]$. Acute intraprocedural thrombus formation during PED procedures remains a significant challenge [10, 11]. This phenomenon is postulated to be due to platelet aggregation because of the high metal density of the device in conjunction with a patient's metabolic hyporesponsiveness to preprocedural dual antiplatelet therapy. Studies attempting to quantify and predict the risk of periprocedural thromboembolic events using the P2Y12 receptor inhibition assay have been inconclusive $[8,10]$. Furthermore, there is no clear consensus or evidence-based protocol for the management of acute thrombus formation during PED embolization cases.

Abciximab (ReoPro; Eli Lilly and Co., Indianapolis, IN, USA), a glycoprotein (GP) IIb/IIIa inhibitor, is utilized as a rescue therapy for thromboembolic complications during intracranial aneurysm coil embolization. Several studies suggest that full cardiac dosing of intraarterial (IA) ReoPro can successfully resolve the acute thrombus and provide flow restoration without increasing the risk of intracranial hemorrhage [12-14]. Experience with ReoPro for acute intraprocedural platelet aggregation in flow diversion is limited, with only two small case series to date $[10,15]$. In each of these series, the delivery method, indication, and dosing algorithm for ReoPro were not standardized. Furthermore, long-term clinical and radiographic outcomes were lacking in these series.

Here, in this report, we identified all neurointerventions where IA ReoPro was administered for the treatment of acute thrombus formation during PED procedures. We hypothesized that a low-dose IA ReoPro bolus, with or without intravenous (IV) maintenance, was sufficient to mitigate periprocedural clinical ischemic complications with a low risk of intracranial hemorrhage.

\section{Methods}

\section{Patient Selection}

This was an institution-based, nonrandomized, retrospective cohort study of a prospectively collected database of intracranial aneurysms treated at a single institution in the USA (The Johns Hopkins Medical Institute, Baltimore, MD, USA). Adult patients treated with the PED and PED Flex (including both anterior and 
posterior circulation aneurysms with and without previous rupture/treatment) from April 2011 to May 2017 were identified in an IRB-approved database. Cases where acute intraprocedural thrombus formation was encountered and treated with ReoPro (IA, IV, or both) were selected for the study.

\section{Data Collection}

Data on patient demographics, aneurysm characteristics, proximal vessel tortuosity (of the cervical internal carotid artery (ICA) and cavernous carotid artery, as previously defined [16, 17]), modified Rankin Scale (mRS) scores (at the time of discharge and at the last follow-up), discharge disposition, PED specifications, dosing of IA ReoPro, dosing of IV ReoPro, the amount of IV heparinization, preoperative antiplatelet agents, and the postprocedural antiplatelet regimen were collected. Periprocedural details including radiographic evaluation of thrombus resolution, radiographic incidence of new ischemia, and/or acute intracranial hemorrhage were recorded. The data are presented as counts, percentages, and means. When means are presented, the standard error of the mean (SEM) is used to assess the sample distribution.

\section{Endovascular Procedure}

The patients were typically treated preoperatively with daily aspirin $325 \mathrm{mg}$ and clopidogrel $75 \mathrm{mg}$ for 7 days. Platelet inhibition testing was not routinely used. Endovascular procedures were performed on a biplanar flat panel angiographic system (Artis Zee; Siemens, Erlangen, Germany) under general anesthesia. Intraprocedural systemic anticoagulation was administered with an initial IV heparin bolus of 5,000 units followed by 1,000 units per subsequent hour. For all cases, a triaxial system (long guide sheath, distal intracranial catheter, and microcatheter) was used through an 8-Fr-short-sheath femoral access. The PED was deployed under real-time visualization using native fluoroscopy (7.5 pulses/s), Road Map, and digital subtraction angiography (DSA; 3 frames/s). Control DSA was performed immediately after deployment as well as 5 and $10 \mathrm{~min}$ after deployment to confirm vessel wall apposition and parent vessel patency and to rule out acute intraluminal thrombi.

If acute thrombus formation was noted on DSA, an IA ReoPro bolus of either $5 \mathrm{mg}$ or $0.125 \mathrm{mg} / \mathrm{kg}$ (half cardiac dose) based on the ideal body weight was directly infused through the triaxial system. Concurrently, either 1,000 or 2,000 units of IV heparin were administered. Control DSA was performed at 5-min intervals after the IA ReoPro bolus. If a significant thrombus persisted on radiographic imaging, an additional 5-mg or $0.125 \mathrm{mg} / \mathrm{kg}$ IA ReoPro bolus was given a maximum of 2 additional times. In cases with complete recanalization after IA ReoPro treatment, IV ReoPro was not used postprocedurally. If a residual thrombus was present after the IA bolus, then IV ReoPro infusion at a rate of $0.125 \mu \mathrm{g} / \mathrm{kg} / \mathrm{min}$ was initiated immediately and continued for $12 \mathrm{~h}$ after the procedure.

After each case, DynaCT without contrast was performed to further assess device opening and to rule out intracranial hemorrhage. After the procedure, all patients were admitted to the neurocritical care unit. The femoral arterial sheath was removed the following day. Daily aspirin $325 \mathrm{mg}$ was continued for the majority of patients. For those patients without medical or logistical contraindications, daily prasugrel (Effient) 5 or $10 \mathrm{mg}$ (based on the body mass index) was initiated and clopidogrel was discontinued. The patients were seen in follow-up at 1 month and 6 and 12 months. Follow-up cerebral angiography was performed at 6 and 12 months.

\section{Results}

During the study period of April 2011 to May 2017, a total of 680 PED/PED Flex procedures were performed for the treatment of intracranial aneurysms. Of these 680 PED cases, only 30 were identified where an acute thrombus had formed intraprocedurally and had been treated with IA ReoPro. The case summaries for these 30 PED procedures are presented in Tables 1 and 2.

\section{Patient and Aneurysm Characteristics}

The mean patient age was 58 years (range $37-85)$, with the majority $(26 / 30 ; 87 \%$ ) of the patients being female. The mean aneurysm size was $8.6 \mathrm{~mm}$ (range 2-25), with small aneurysms $(<10 \mathrm{~mm})$ accounting for $63 \%(19 / 30)$ of the cases. Most of the aneurysms $(27 / 30$; 
Table 1. Patient demographics and aneurysm characteristics

\begin{tabular}{lc} 
Demographics & \\
Total cases & 30 \\
Mean age \pm SD (range), years & $56.7 \pm 11(36-84)$ \\
Female sex & $26(87)$ \\
Previously treated & $5(17)$ \\
Clip & $0(0)$ \\
Coil & $4(13)$ \\
Flow diversion & $1(3)$ \\
\hline Aneurysm characteristics & \\
Total aneurysms & 30 \\
Average size \pm SD (range), mm & $8.6 \pm 5.7(2-25)$ \\
Aneurysm size & \\
Small (<10 mm) & $19(63)$ \\
Large (10-25 mm) & $11(37)$ \\
Giant (>25 mm) & 0 \\
Anterior circulation & $27(90)$ \\
Internal carotid & $23(77)$ \\
$\quad$ Cavernous & $5(17)$ \\
Ophthalmic/paraophthalmic & $10(33)$ \\
Supraclinoid/PCOM & $6(20)$ \\
Termination & $2(7)$ \\
Anterior cerebral & $3(10)$ \\
A1 & $1(3)$ \\
A1-2/A2-3 & $2(7)$ \\
Middle cerebral & $1(3)$ \\
Bifurcation & $1(3)$ \\
Posterior circulation & $3(10)$ \\
Superior cerebellar artery & $2(7)$ \\
Basilar apex & $1(3)$ \\
\hline & \\
&
\end{tabular}

Values are presented as $n(\%)$ unless stated otherwise. PCOM, posterior communicating.

90\%) were in the anterior circulation. Internal carotid artery aneurysms along the ophthalmic/ paraophthalmic segment were the most common (10/30; 33\%). Only 3 cases were posterior circulation aneurysms ( 2 in the superior cerebellar artery and 1 in the basilar apex). Five cases were previously treated aneurysms ( 4 coiled and 1 flow diverted), with subsequent residuals/recurrence requiring additional treatment.

\section{Proximal Vascular Access Characteristics}

Of the 27 cases of anterior circulation aneurysm, 12 (44\%) had significant cervical ICA tortuosity and $11(41 \%)$ had moderate-to-severe cavernous ICA tortuosity characterized by a cavernous ICA grade of II-IV [17]. The 0.058" AXS Catalyst 5 (Stryker Neurovascular, Fremont, CA, USA) and 0.058" Navien (Medtronic Neurovascular) were the most frequently utilized distal intracranial catheters. The most common distal intracranial catheter position was along the cavernous segment of the ICA. Verapamil infused intra-arterially was used in 5 $(17 \%)$ of the cases to treat catheter-induced vasospasm.

\section{Procedural Details}

All 30 cases were completed with successful PED/PED Flex implantation. The average fluoroscopy time was $61 \pm 34 \mathrm{~min}$, with an average radiation exposure of 3,682 $\pm 1,695 \mathrm{mGy}$. No intraprocedural rupture was encountered. The average number of PEDs implanted per case was 1.2. In 10 cases, adjunctive coiling was performed (range 1-15 coils). Balloon angio- 
Table 2. Procedural details

\begin{tabular}{|c|c|}
\hline Immediate complete or partial resolution of thrombosis & $30(100 \%)$ \\
\hline \multicolumn{2}{|l|}{ Access, catheters, and anatomy } \\
\hline Significant cervical ICA tortuosity & $12 / 27(44 \%)$ \\
\hline \multicolumn{2}{|l|}{ Cavernous ICA grade (27 anterior circulation cases) } \\
\hline Ia & $5(18 \%)$ \\
\hline $\mathrm{Ib}$ & $11(41 \%)$ \\
\hline II & $5(18 \%)$ \\
\hline III & $3(11 \%)$ \\
\hline IV & $3(11 \%)$ \\
\hline \multicolumn{2}{|l|}{ Distal intracranial catheter used } \\
\hline Navien $0.058^{\prime \prime}$ & $8(27 \%)$ \\
\hline Navien $0.072^{\prime \prime}$ & $4(13 \%)$ \\
\hline Catalyst $50.058^{\prime \prime}$ & $14(47 \%)$ \\
\hline Sofia & $2(7 \%)$ \\
\hline InNeuroCo $0.058^{\prime \prime}$ & $2(7 \%)$ \\
\hline \multicolumn{2}{|l|}{ Distal intracranial catheter position } \\
\hline Cervical & $0(0 \%)$ \\
\hline Petrous & $3(10 \%)$ \\
\hline Proximal cavernous & $14(47 \%)$ \\
\hline Distal cavernous & $8(27 \%)$ \\
\hline Supraclinoid & $1(3 \%)$ \\
\hline $\mathrm{A} 1$ & $1(3 \%)$ \\
\hline V2-4 & $3(10 \%)$ \\
\hline \multicolumn{2}{|l|}{$0.027^{\prime \prime}$ microcatheter used } \\
\hline Marksman & $12(40 \%)$ \\
\hline VIA & $18(60 \%)$ \\
\hline \multicolumn{2}{|l|}{ Case characteristics } \\
\hline Average fluoroscopy time \pm SD (range), min & $61 \pm 34(21-152)$ \\
\hline Average radiation exposure \pm SD (range), $\mathrm{mGy}$ & $3,682 \pm 1,695(1,418-7,266)$ \\
\hline Adjunctive coiling & $10(33 \%)$ \\
\hline Vasospasm (verapamil infusion) & $5(17 \%)$ \\
\hline Average number of PEDs implanted per case \pm SD (range) & $1.2 \pm 0.6(1-3)$ \\
\hline Balloon angioplasty & $11(37 \%)$ \\
\hline Intraprocedural rupture & $0(0 \%)$ \\
\hline PED cork/removal & $2(7 \%)$ \\
\hline Intracerebral hemorrhage postprocedurally & $2(7 \%)$ \\
\hline Ischemic infarcts postprocedurally & $4(13 \%)$ \\
\hline
\end{tabular}

ICA, internal carotid artery; PED, Pipeline embolization device.

plasty to postprocess the deployed PED was utilized in $11(37 \%)$ of the cases to improve vessel wall apposition.

Details on the dual antiplatelet regimen, periprocedural ReoPro dosing, and clinical follow-up are presented in Table 3 . All but 2 cases were preoperatively treated with daily aspirin $325 \mathrm{mg}$ and clopidogrel $75 \mathrm{mg}$ for 7 days: one case with a history of subarachnoid hemorrhage 3 months prior to PED embolization (case No. 3) where aspirin only was used, and the other case with known clopidogrel intolerance where ticagrelor was used instead (case No. 18).

Increments of 5-mg IA ReoPro boluses were administered in 20 cases, while increments of $0.125 \mathrm{mg} / \mathrm{kg}$ boluses (half cardiac dose) were given in 10 cases. Immediate complete or partial resolution of the acute thrombosis was observed in $100 \%$ of the cases. Case No. 8 serves as an example of where a single dose of $5 \mathrm{mg}$ IA ReoPro resulted in immediate, complete recanalization (Fig. 1). In 22 cases with partial recanalization after IA ReoPro treatment, postproce- 
Lin et al.: Abciximab (ReoPro) Dosing Strategy in Pipeline Flow Diversion Treatment of Intracranial Aneurysms

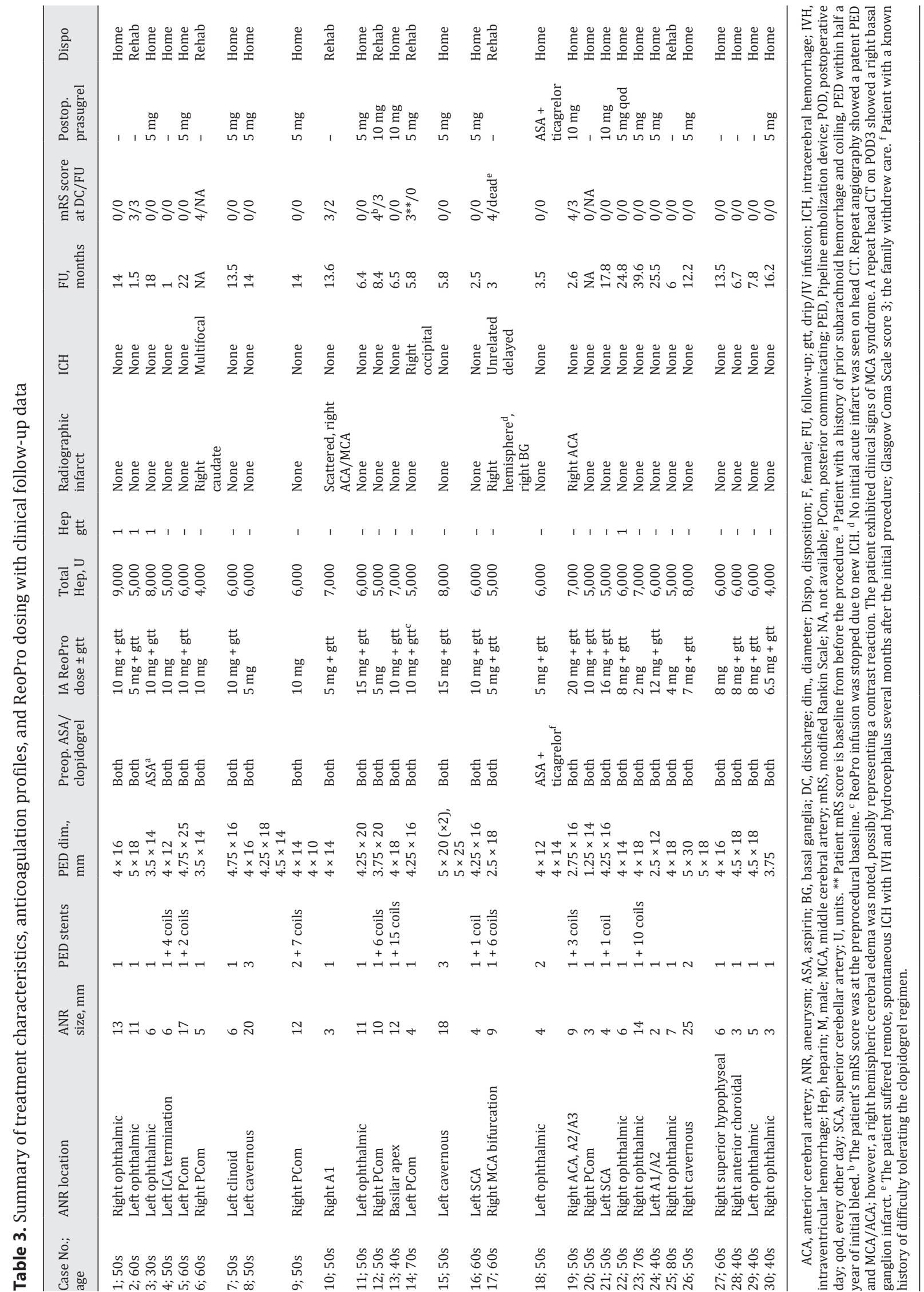




\section{Neurology}

Lin et al.: Abciximab (ReoPro) Dosing Strategy in Pipeline Flow Diversion Treatment of Intracranial Aneurysms
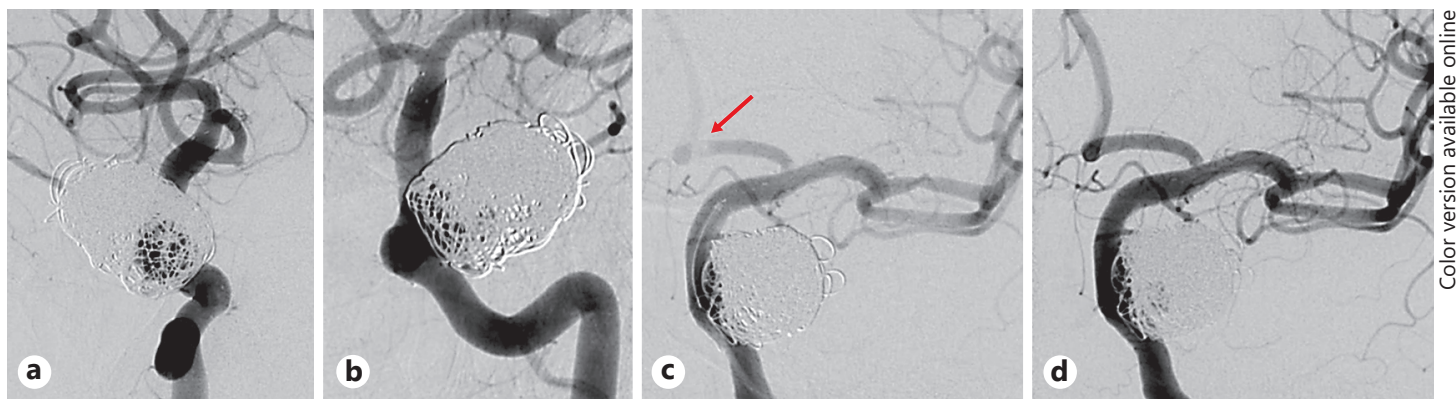

Fig. 1. Complete recanalization of in situ thrombosis in patient No. 8 after a 5-mg IA ReoPro bolus. a, b Left internal carotid digital subtraction angiogram, lateral view (a) and anteroposterior view (b), showing patient No. 8 with a 20-mm left cavernous internal carotid artery aneurysm with previous Neuroform stent-assisted coil embolization with recurrent filling on cerebral angiography. c Left internal carotid digital subtraction angiogram, oblique view. Immediate post-deployment angiography following deployment of the third Pipeline embolization device demonstrating a filling defect at the left A1 orifice and possibly the left A1/2 junction (arrow). d Left internal carotid digital subtraction angiogram, oblique view. Complete recanalization seen following the administration of $5 \mathrm{mg}$ IA ReoPro.

dural IV ReoPro infusion was administered (Fig. 2). In 4 cases (treated in the early years 20112013), systemic IV heparin was also given after the procedure in addition to the IV ReoPro. After 2013, postprocedural IV heparin infusion was not used. In 18 cases, postprocedural dual antiplatelet regimens were transitioned to prasugrel (Effient)/aspirin instead of the preprocedural clopidogrel/aspirin regimens. The patients who did not transition to prasugrel from clopidogrel had contraindications including limitations with insurance reimbursement (case No. 10), concerns about a high bleeding risk with excessive bruising (case No. 27), and/or other medical comorbidities such as active gastrointestinal bleeds (case No. 25) or end-stage renal disease undergoing transplant evaluation with known coagulopathy (case No. 4).

\section{Ischemic or Hemorrhagic Complications}

In the majority $(23 / 30 ; 77 \%)$ of the cases, the patients were discharged home on either postoperative day (POD) 1 or 2 . At the time of discharge, 23/30 (77\%) had an mRS score of 0 , $3 / 30$ had an mRS score of 3 ( 1 was baseline preprocedurally), and 4/30 had an mRS score of 4 ( 1 was baseline preprocedurally). Of the 5 patients with a discharge $\mathrm{mRS}$ score $>0$, without any preexisting disability at baseline, only 4 (cases No. 6, 10,17, and 19) had ischemic infarcts (Fig. 3).

Intracranial hemorrhage occurred in only 2 cases (7\%) (Fig. 4). Case No. 6 was a sexagenarian with a previously coiled, 5-mm, ruptured right posterior communicating artery aneurysm. During PED treatment of aneurysm recurrence, in-stent thrombosis and subsequent flow limitation in the right anterior cerebral artery (ACA) was noted. A total of $10 \mathrm{mg}$ IA ReoPro was administered, with marked resolution of the thrombus and recanalization of the right A1 segment as well as the distal ACA territory. A postprocedural routine head CT revealed multifocal intracerebral hemorrhage (ICH), largest in a hypodense region of the right caudate head, likely representing hemorrhagic transformation of an acute infarction. Clopidogrel was maintained for 3 days, while daily aspirin $325 \mathrm{mg}$ was continued. The patient was subsequently discharged to a rehabilitation facility on POD4 with an mRS score of 4 and has subsequently been lost to follow-up. Case No. 14 was a septuagenarian who presented with a Glasgow Coma Scale score of 10 with a ruptured, 4-mm left posterior communicating artery aneurysm which was treated at the time with coil embolization. The patient was eventually discharged to a rehabilitation facility with an mRS score of 3. Follow-up angiography showed a residual aneurysm 


\section{Interventional Neurology}

\begin{tabular}{l|l}
\hline Intervent Neurol 2018;7:218-232 \\
\hline DOI: 10.1159/000486458 & $\begin{array}{l}\text { @ 2 2018 S. Karger AG, Basel } \\
\text { www.karger.com/ine }\end{array}$ \\
\hline
\end{tabular}

Lin et al.: Abciximab (ReoPro) Dosing Strategy in Pipeline Flow Diversion Treatment of Intracranial Aneurysms
Fig. 2. Partial recanalization of instent thrombosis in case No. 1 after a 10-mg IA ReoPro bolus and systemic IV ReoPro infusion. a Digital subtraction angiogram (lateral view) of the right internal carotid artery (ICA), illustrating a 13-mm right ophthalmic segment aneurysm. b Native fluoroscopy image, lateral view, demonstrating the deployment of a $4 \times 16 \mathrm{~mm}$ Pipeline embolization device (PED) across the neck of the aneurysm. c-e Right ICA digital subtraction angiogram, lateral view, illustrating the deployment of the PED and immediate control angiography. c Aneurysmal stasis is demonstrated with an eclipse sign during final PED deployment, as well as significant atherosclerosis and mild speckling near the proximal margins of the device. d Following retrieval of the PED delivery wire, digital subtraction angiography demonstrated significant lucent speckling throughout the PED construct and limited opacification of the right anterior cerebral artery. e Following $10 \mathrm{mg}$ of IA ReoPro, right ICA injection demonstrates stabilization of the thrombus and improved flow distal to the PED. The patient was subsequently placed on an IV ReoPro infusion overnight at $0.125 \mu \mathrm{g} / \mathrm{kg} / \mathrm{min}$.
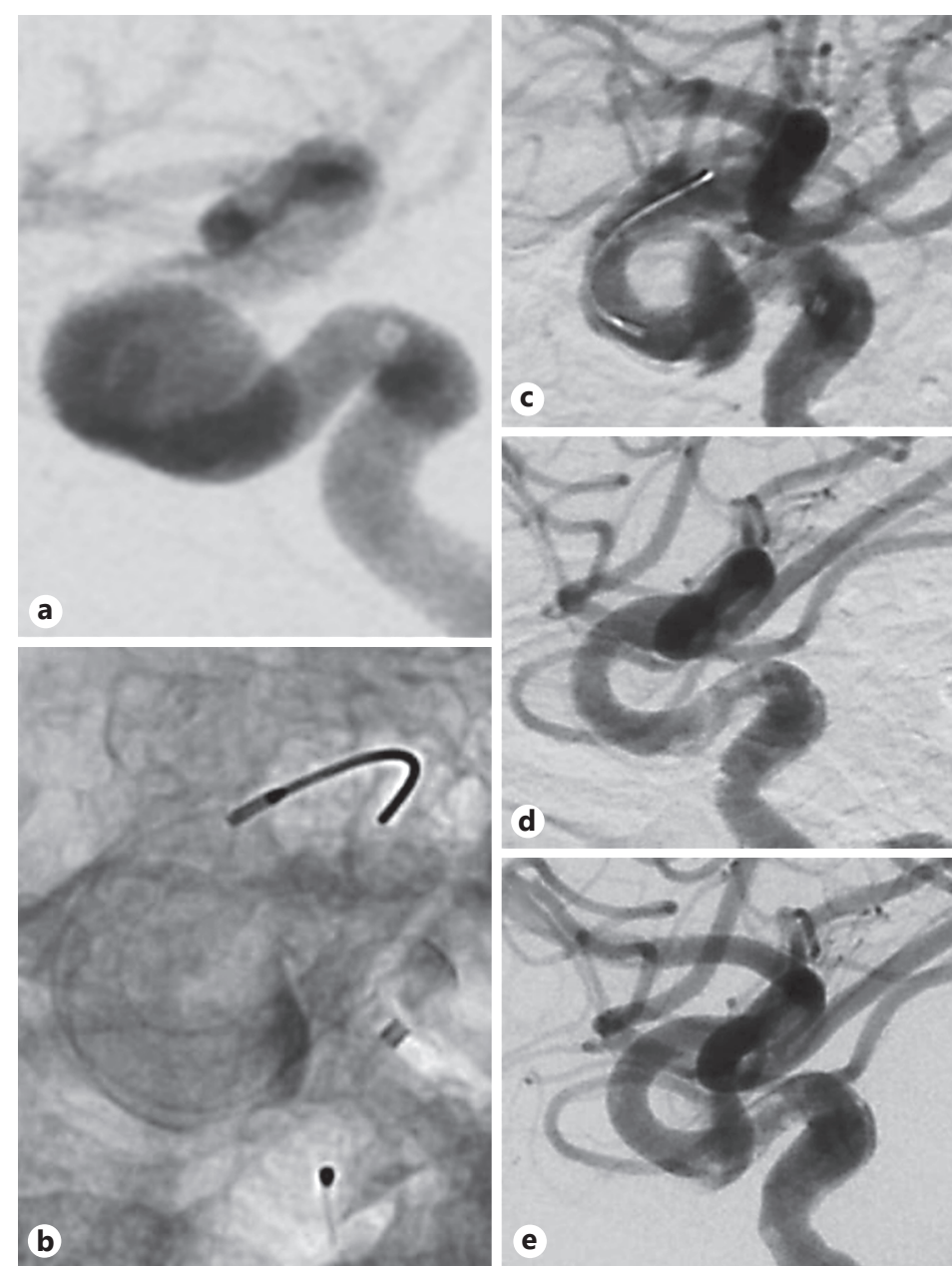

in the neck for which PED treatment was recommended. During PED deployment, device malposition was noted, requiring balloon angioplasty to improve vessel wall apposition. After the angioplasty procedure, in-stent thrombosis developed. A total of 10 mg IA ReoPro was administered with partial recanalization. IV ReoPro infusion was initiated after the procedure. The patient subsequently developed left upper extremity weakness, and a head CT revealed a $16-\mathrm{cm}^{3}$ right parietal-occipital ICH. The patient's IV ReoPro infusion was maintained and an operative intervention was not necessary. The patient was discharged to rehabilitation on POD6 with a stable mRS score of 3. By the last follow-up at 5.8 months, the patient had completely recovered with an mRS score of 0 and received medical clearance to drive a motor vehicle.

\section{Long-Term Clinical Follow-Up}

Twenty-eight patients underwent clinical follow-up for an average of 11.7 months. At the last clinical follow-up, 23/28 (82\%) of the patients had an mRS score of 0 . Of the 5 patients with a follow-up mRS score $>0,4$ had a stable or improved $\mathrm{mRS}$ score when compared to their discharge score. For example, Figure 5 illustrates case No. 19, a quinquagenarian who 


\section{Interventional Neurology}
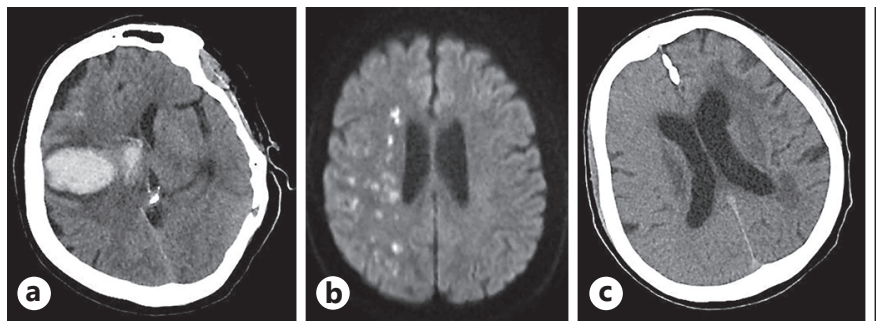
Intracranial Aneurysms

Lin et al.: Abciximab (ReoPro) Dosing Strategy in Pipeline Flow Diversion Treatment of

Fig. 3. Radiographic presentation of 4 cases (No. 6, 10,17, and 19) with ischemic infarcts. a Axial noncontrast head CT of patient No. 6 demonstrating right caudate ischemia with hemorrhagic conversion. b Diffusionweighted image of patient No. 10 showing scattered infarcts in the right anterior cerebral artery (ACA)/ middle cerebral artery subcortical and watershed regions. c, d Axial noncontrast head CT (c) of patient No. 17 showing a right hemispheric cerebral edema and loss of gray/white differentiation on postoperative day (POD) 1, with improved edema but a new right basal ganglion infarct on POD3 (d). e Diffusion-weighted image showing patient No. 19 with right ACA cortical and subcortical scattered infarcts.

underwent coil-assisted PED treatment of a 9-mm right A2-3 ACA aneurysm, complicated by in-stent and pericallosal artery thrombosis. A total of $20 \mathrm{mg}$ IA ReoPro was administered with near-complete radiographic resolution of the thrombus. The patient was maintained on IV ReoPro infusion postprocedurally, but was noted to have new-onset left-sided weakness. Head CT, CT angiography, and repeat diagnostic angiography on POD1 did not reveal any large vessel occlusion, in-stent thrombosis, or acute hemorrhage. The patient's weakness persisted, and a follow-up MRI demonstrated multiple small infarcts in the right ACA subcortical and watershed territory. The patient was managed expectantly and was discharged with an $\mathrm{mRS}$ score of 4 , unable to ambulate. By the 2.6-month follow-up, the patient had regained ambulatory function with an mRS score of 3 .

One delayed death was encountered in this series. Case No. 17 was a sexagenarian with chronic kidney disease and congestive heart failure found incidentally with a 9-mm, irregularly contoured right middle cerebral artery (MCA) bifurcation aneurysm. During coil-assisted PED embolization, a small platelet plug was visualized in the proximal portion of the device and $5 \mathrm{mg}$ of IA ReoPro was administered with subsequent improvement. Postprocedurally, the patient had grade 0-1 strength (out of 5) in the left upper and lower extremities. Repeat angiography showed a widely patent right MCA and PED construct. Head CT on POD1 and 2 showed a diffuse cerebral edema in the right hemisphere consistent with a contrast reaction without infarction. Head CT on POD3 demonstrated a small right basal ganglion infarct. The patient was discharged to a rehabilitation facility with an mRS score of 4, then was briefly admitted 3 months later for seizures and a negative radiographic workup. Unfortunately, only 4 days after that hospitalization, the patient was found unresponsive and head CT revealed a large left basal ganglion ICH with significant intraventricular hemorrhage and hydrocephalus. This injury was on the contralateral side of the prior procedure and was likely a hemorrhagic conversion of a new infarct. Considering the patient's poor neurological prognosis, the family elected for comfort care and the patient expired from this unrelated injury.

\section{Discussion}

Thromboembolic complications are known risks of endovascular treatment for cerebral aneurysms, with reported rates ranging from 2 to $15 \%$ for coiling alone, and from 4 to $20 \%$ for stent-assisted coiling and flow diversion $[18,19]$. Dual antiplatelet therapy is routinely 


\section{Interventional Neurology}

Intervent Neurol 2018;7:218-232

\begin{tabular}{l|c|}
\hline DOI: 10.1159/000486458 & (c) 2018 S. Karger AG, Basel
\end{tabular}

www.karger.com/ine

Lin et al.: Abciximab (ReoPro) Dosing Strategy in Pipeline Flow Diversion Treatment of Intracranial Aneurysms
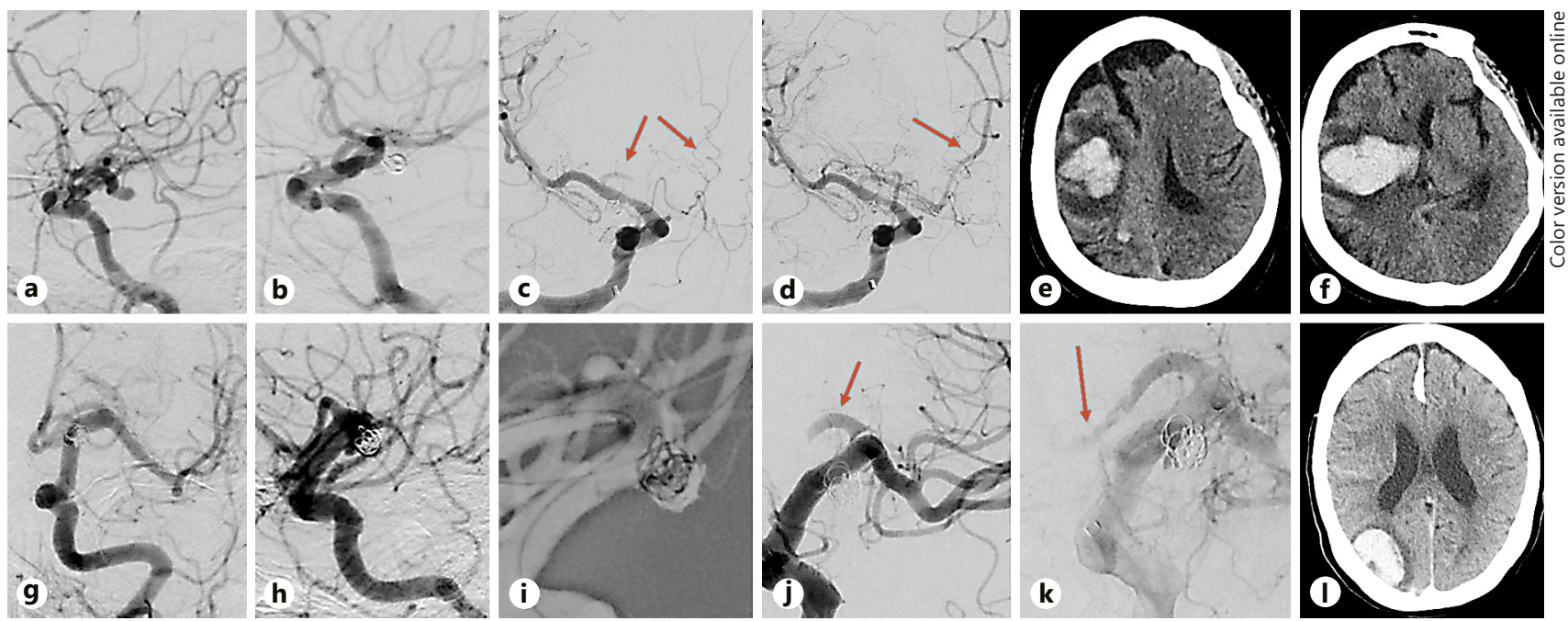

Fig. 4. Radiographic presentation of patients No. 6 (a-f) and No. 14 (g-l) with hemorrhagic complications. a, b Right internal carotid artery (ICA) digital subtraction angiograms, lateral oblique view, demonstrating a 5-mm right posterior communicating artery (PCOM) aneurysm in patient No. 6 before (a) and after coiling (b). c Right ICA digital subtraction angiogram, anteroposterior (AP) view, showing an intraprocedural thrombus and flow limitation in the right anterior cerebral artery (ACA) (arrows). d Right ICA digital subtraction angiogram, AP view, showing complete recanalization of the right ACA following ReoPro administration (arrow). e, f Axial noncontrast head CT demonstrating right frontal and right internal capsule intracranial hemorrhage (ICH). g, h Left ICA digital subtraction angiograms, AP (g) and lateral view (h), demonstrating persistent filling of a previously coiled 4-mm left PCOM aneurysm in patient No. 14. i Left ICA native fluoroscopy, oblique view. Balloon angioplasty using a $5 \times 10 \mathrm{~mm}$ Transform balloon for small-segment wall malapposition. j, k Left ICA digital subtraction angiogram (j) and native fluoroscopy image (k), AP view, after angioplasty showing in situ thrombosis and partial recanalization following $10 \mathrm{mg}$ IA ReoPro (arrows). I Axial noncontrast head CT showing postprocedural right occipital ICH.
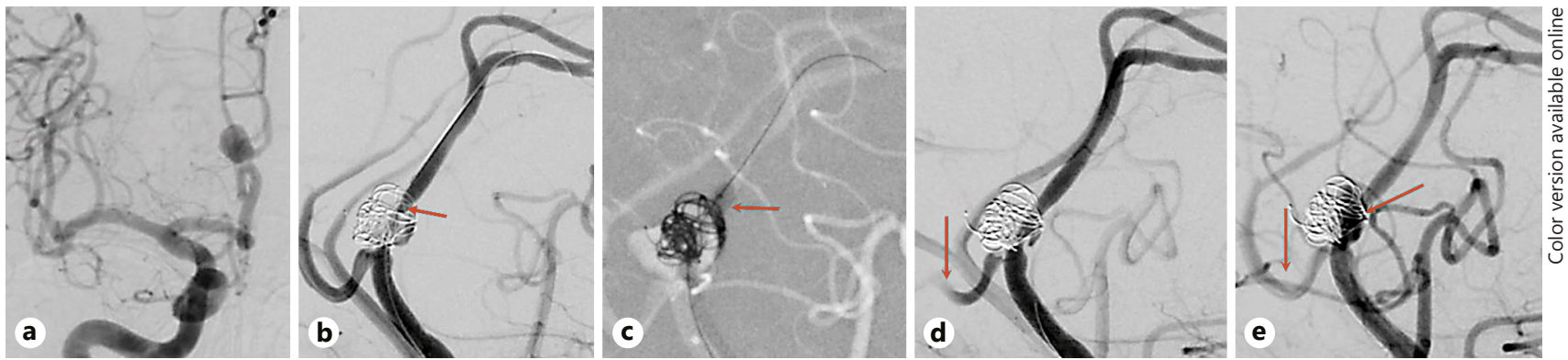

Fig. 5. Partial recanalization of in situ thrombosis in patient No. 19 after a 20-mg IA ReoPro bolus, necessitating IV ReoPro infusion. a Right internal carotid artery (ICA) digital subtraction angiogram, anteroposterior (AP) view, showing patient No. 19 with an incidentally discovered, 9-mm right A2-3 bifurcation aneurysm with morphological irregularity. b Right ICA digital subtraction angiogram, AP view, showing platelet aggregation within the proximal portion of the pipeline stent (arrow) causing partial occlusion of the pericallosal artery; 10 mg of IA ReoPro was administered through the Catalyst 5 catheter. c Right ICA Road Map, AP view. A $4 \times 7$ Transform SC balloon was used for angioplasty (arrow). d Right ICA digital subtraction angiogram, AP view, demonstrating recurrence of platelet aggregation within the callosal marginal artery; thus, an additional 10 mg of IA ReoPro was administered. e Right ICA digital subtraction angiogram, AP view, demonstrating near-complete recanalization of both the pericallosal and callosal marginal arteries after $20 \mathrm{mg}$ IA ReoPro; subsequently, IV ReoPro infusion was initiated at $0.125 \mu \mathrm{g} / \mathrm{kg} / \mathrm{min}$. 
prescribed periprocedurally to mitigate this ischemic infarct risk during stent-assisted coiling and flow diversion treatments. Due to the higher metal density of flow diverters compared to the traditional intracranial stents for coiling, adjunct preprocedural platelet inhibition testing was proposed to assist with thromboembolic risk stratification, but this remains a controversial subject within the flow diversion literature $[8,10]$. A recent retrospective multicenter study by Adeeb et al. [20] defined clopidogrel nonresponders as having a P2Y12 level >208 and concluded that the rate of thromboembolic complications in PED cases for this group was $17.4 \%$ versus $5.6 \%$ for the clopidogrel responders (P2Y12 reaction units [PRU] <208). In contrast, Bender et al. [8] evaluated a series of 52 patients with a P2Y12 level $>200$ undergoing PED treatment for intracranial aneurysms and found the rate of thromboembolic complications to be only $4 \%$, concluding that this "nonresponder" level was not associated with increased thromboembolic complications in anterior circulation PED treatments. While a consensus on preembolization antiplatelet dose titration and platelet inhibition testing for PED procedures is not imminent, rapid rescue therapy for intraprocedural platelet plug and thrombus formation is an essential tool for the neurointerventionalist in not only PED treatments but any intracranial stent procedures.

Abciximab (ReoPro; Eli Lilly) is the most widely used GP IIb/IIIa inhibitor for rescue therapy in the endovascular literature. GP IIb/IIIa inhibitors act by preventing fibrinogen from binding to the GP IIb/IIIa receptor, thus preventing platelet activation, aggregation, and plug/thrombus formation. ReoPro is a monoclonal human antibody with a high receptor affinity and long duration of action. Initial experience with ReoPro began in the cardiac field, and it has long been established with a dosing regimen of IV ReoPro boluses of $0.25 \mathrm{mg} / \mathrm{kg}$ followed by an infusion rate of $0.125 \mu \mathrm{g} / \mathrm{kg} / \mathrm{min}[21,22]$. In the neuroendovascular coil embolization literature, the dosing and administration regimens for ReoPro have been heterogeneous and extremely variable [12-14]. Some studies utilized 2-5 mg IA incremental boluses [23, 24], others used a full cardiac loading dose at $0.25 \mathrm{mg} / \mathrm{kg}$ (IA or IV) [13,25], and some used a combination of the above with continuous IV infusion after the initial IA bolus $[26,27]$. A review of the key studies on this topic was aptly performed by Dornbos et al. [28]. The authors summarized that despite the high variability in dosing and administration regimens, the overall recanalization rates were $72-100 \%$, with rates of ICH as low as $2-3 \%$ $[12-14,23,24,26,28,29]$ and as high as $18 \%$, with $44 \%$ mortality [30]. Dornbos et al. [28] recommend a ReoPro dosing strategy of an initial $0.25 \mathrm{mg} / \mathrm{kg}$ (full cardiac dose) bolus, given IA or IV, followed by continuous IV infusion of $0.125 \mu \mathrm{g} / \mathrm{kg} / \mathrm{min}$ maintained for $12 \mathrm{~h}[28,30]$.

The experience with ReoPro administration in the flow diversion literature for the management of intraprocedural acute thrombus formation is quite limited, with only 2 small case series to date $[10,15]$. In the series by Patel et al. [10], 2 patients received half the cardiac weight-based loading dose and 3 patients received the full dose. All 5 patients had immediate recanalization; therefore, postprocedural IV maintenance was not administered. All 5 patients had an mRS score of 0 at discharge and follow-up. Interestingly, the group noted that 4 of the 5 patients had a P2Y12 value $>200$. In the series by Lall et al. [15], 3 patients with acute thrombosis after PED deployment were treated with the full cardiac $0.25 \mathrm{mg} / \mathrm{kg}$ loading bolus and had prompt recanalization with no postprocedural clinical complications.

Compared to the above studies, our case series is on par with the coil embolization literature with respect to the rate of hemorrhagic and ischemic complications ( 7 and 13\%, respectively). Our clinical outcomes are similar to those in the PED series by Patel et al. [10] and Lall et al. [15], achieving immediate complete or partial recanalization in all 30 cases. Additionally, all but 1 patient in our series had a follow-up mRS score that was stable or improved compared to discharge, and $23 / 28(82 \%)$ had an mRS score of 0 at the last follow-up. Our dosing strategy mirrored the coiling literature in 20 cases where we used increments of 5-mg IA boluses. For 10 cases, we used incremental $0.125 \mathrm{mg} / \mathrm{kg}$ IA loading boluses, which is half the commonly 


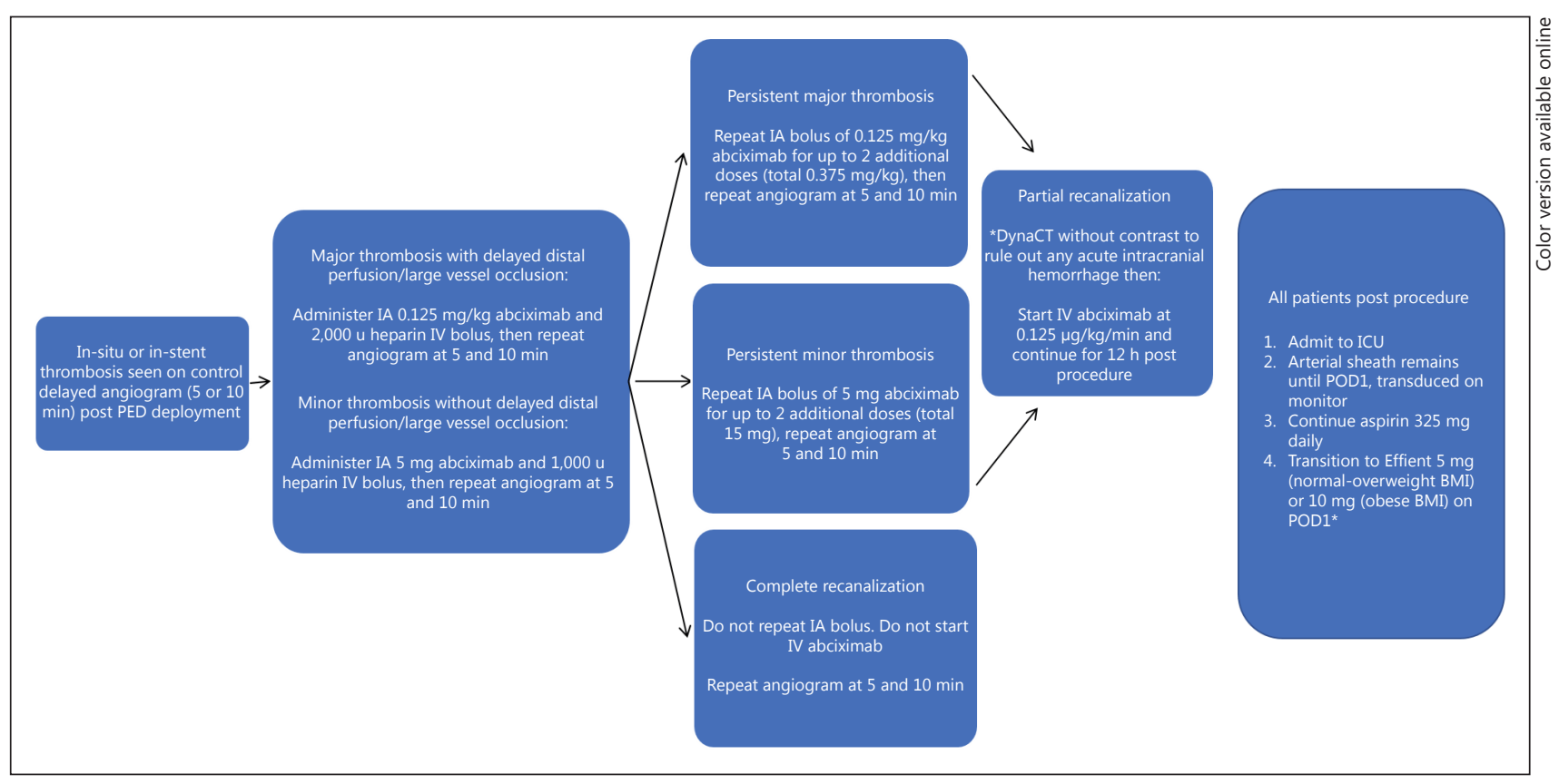

Fig. 6. Rescue protocol for IA and IV ReoPro administration in acute intraprocedural thrombus management in PED embolization neurointerventions. PED, Pipeline embolization device; POD, postoperative day. ${ }^{*}$ Relative contraindications to Effient or Brilinta include: insurance/logistical/cost issues, persistently low P2Y12 reaction units preoperatively and postoperatively, and/or other medical comorbidities such as active bleeding, liver cirrhosis, ESRD, or coagulopathy.

utilized $0.25 \mathrm{mg} / \mathrm{kg}$ dose found in the coil embolization and cardiac literature. In our weightbased loading bolus group, no ischemic or hemorrhagic complications were encountered. Interestingly, the 2 hemorrhagic complications in our series occurred in the 5-mg-increment group, with 2 patients who had recent subarachnoid hemorrhage. Of note, the 5-mg IA bolus dose is a lower dose than the $0.125 \mathrm{mg} / \mathrm{kg}$ loading dose based on an average body weight of $70 \mathrm{~kg}$ (which would entail $8.75 \mathrm{mg}$ ). Our data suggest that starting with a lower loading IA bolus dose than commonly reported in the literature may be sufficient for thrombus resolution and recanalization, without increasing the rate of postprocedural ischemic infarcts, and minimizing the hemorrhagic risk.

We have since strategized a standardized protocol for dosing IA and IV ReoPro rescue treatment of intraprocedural acute thrombus formation in PED embolization procedures. Figure 6 illustrates this protocol, which includes the imaging details needed to evaluate thrombus resolution, the timing of IV ReoPro infusion, and nuances of postprocedural care including femoral sheath management and an antiplatelet strategy. Systemic heparin infusion was utilized in the infancy of this experience (2011-2013), when the ReoPro literature in the neuroendovascular field was still in the early stages. To avoid potential hemorrhagic complications in cases of partial recanalization, we no longer use dual IV heparin and ReoPro infusion. To rapidly and urgently implement the protocol outlined in Figure 6, the neuroendovascular suite Pyxis must have ReoPro stocked and readily accessible. Postprocedurally, all patients in our series were admitted to a dedicated neurocritical care unit for intensive monitoring. We also prefer maintaining femoral arterial sheath placement overnight, as its immediate removal following ReoPro administration can lead to increased risks for groin hematoma and retroperitoneal hemorrhage. Additionally, an indwelling sheath in the acute postoperative period provides rapid access to repeat angiography if clinically indicated. We 
connect the femoral sheath to a transducer, while an arterial line waveform is displayed on the monitor as a safety measure. Given both the inconclusive P2Y12 literature and the fact that the assay results are affected by ReoPro administration, we transition patients' antiplatelet regimen from aspirin and clopidogrel dual therapy to aspirin and another adenosine diphosphate receptor inhibitor (prasugrel, ticlopidine, or ticagrelor) unless medical or logistical contraindications exist. On postprocedural day 1, we routinely start $5 \mathrm{mg}$ of prasugrel (Effient) for patients with a normal weight to overweight, and $10 \mathrm{mg}$ prasugrel for patients with a BMI in the obese range, sans loading dose, to be continued as an outpatient.

During the study time period of April 2011 to May 2017, a total of 680 PED embolization procedures were performed at our institution. All patients were preoperatively treated with daily aspirin $325 \mathrm{mg}$. For the second antiplatelet, daily clopidogrel $75 \mathrm{mg}$ was used in 670 cases, daily prasugrel 5 or $10 \mathrm{mg}$ (weight based) was used in 8 cases, and ticagrelor $90 \mathrm{mg}$ twice daily was used in 2 cases. Only 30 of the 680 PED cases developed acute thrombus formation intraprocedurally, with a calculated in situ thrombosis risk of $4.4 \%$. Furthermore, of this 30-case subgroup with intraprocedural acute thrombus formation, only 4 were complicated by ischemic infarcts. Interestingly, 3 of these 4 cases were treatments for aneurysms located beyond the ICA ( 2 in the ACA and 1 in the MCA). Considering that only a fraction (4/680 total; $0.6 \%$ ) of these patients suffered an embolic complication, this observed rate is superior to the $3-6 \%$ risk of thromboembolic complications for PED treatments reported in previous studies $[1,8,9]$. We have previously reported a similar rate of thromboembolic complications in patients with high P2Y12 levels (PRU >200), considered as "nonresponders" [8]. As such, we continue to strongly caution against preprocedural titration of antiplatelet medications to P2Y12 levels, given the likelihood of increasing hemorrhagic risks with this practice.

ReoPro is the GP IIb/IIIa inhibitor of choice for rescue therapy at our institution. Eptifibatide (Integrilin), an alternative GP IIb/IIIa inhibitor, is preferentially used at various centers. In comparison to ReoPro, eptifibatide is a small-molecule GP IIb/IIIa inhibitor with a shorter pharmacokinetic half-life, which allows for rapid return of platelet function in the event of hemorrhagic complications. Ramakrishnan et al. [31] utilized incremental loading doses starting at $25 \%$ of a $90 \mu \mathrm{g} / \mathrm{kg}$ IA bolus, followed by $0.5 \mu \mathrm{g} / \mathrm{kg} / \mathrm{min}$ IV infusion for 20 aneurysm patients, with a $97.5 \%$ complete or partial recanalization rate and $0 \%$ intracranial hemorrhage. Similarly, Sedat et al. [32] reported a 91\% recanalization rate, without any incidence of intracranial hemorrhage, in a 45-patient aneurysm cohort using a single IA $0.2 \mathrm{mg} /$ kg eptifibatide bolus. The review by Dornbos et al. [28] of GP IIb/IIIa inhibitor use in endovascular treatments of aneurysms recommends using a $90 \mu \mathrm{g} / \mathrm{kg}$ IA bolus, followed by 0.5 $\mu \mathrm{g} / \mathrm{kg} / \mathrm{min} \mathrm{IV}$ infusion for $12 \mathrm{~h}$ for rescue therapy. The protocol established in this study uses the lowest effective bolus of IA ReoPro followed by IV infusion, if necessary, and can similarly be applied to eptifibatide administration.

The limitations of the current study are due to its retrospective, single-institution nature. There is also heterogeneity in ReoPro dosing within our series. As a result, our recommendations for best practice guidelines are as specified in Figure 6. Prospective, randomized studies will be required to establish further protocols.

\section{Conclusion}

IA ReoPro administration is an effective and safe strategy for the management of acute in situ thrombogenic complications during PED treatment. Remarkably, high rates of immediate thrombus resolution can be achieved with half the cardiac and coil embolization bolus dosage $(0.125 \mathrm{mg} / \mathrm{kg})$ or even less. We recommend incremental IA boluses of $0.125 \mathrm{mg} / \mathrm{kg}$ for major acute thrombus formation, and 5-mg increment boluses for minor thrombi. Post- 
procedural IV ReoPro infusion is necessary in cases where IA boluses of ReoPro result only in partial recanalization. Such a careful dose escalation methodology provides relatively low rates of hemorrhagic complications with high recanalization rates, and yields optimal longterm clinical outcomes.

\section{Statement of Ethics}

All individual identifying information, including age and sex, have been meticulously anonymized to achieve appropriate anonymity in the paper.

\section{Disclosure Statement}

A.L.C. is a proctor for the Woven EndoBridge device (Sequent Medical, Aliso Viejo, CA, USA), a proctor for the Surpass device (Stryker Neurovascular), a consultant for Stryker Neurovascular, a proctor for the PED (Medtronic Neurovascular), a consultant for Medtronic, a proctor for the FRED device (MicroVention, Tustin, CA, USA), and a consultant for MicroVention-Terumo and InNeuroCo. G.P.C. is a consultant for both Medtronic and MicroVention-Terumo, and participates in clinical trials for Medtronic and Stryker Neurovascular. L.-M.L. is a proctor for the PED (Medtronic Neurovascular) and a consultant for MicroVention-Terumo, and participates in clinical trials for Stryker Neurovascular. The other authors have no conflict of interest to disclose. No author received financial support for the generation of this paper.

\section{Author Contributions}

L.-M.L., G.P.C., B.J., J.K.C., and N.B.B. drafted the manuscript and critically revised it for important intellectual content. M.T.B., J.K.C., and B.J. assisted with the data collection and analysis. G.P.C. performed treatment procedures and assisted in critically revising the manuscript. M.T.B., J.H., and R.J.T. crucially reviewed the important intellectual content of the manuscript. A.L.C. performed treatment procedures and critically revised the important intellectual content. All authors read and approved the final manuscript.

\section{References}

1 Kallmes DF, Hanel R, Lopes D, Boccardi E, Bonafé A, Cekirge S, Fiorella D, Jabbour P, Levy E, McDougall C, Siddiqui A, Szikora I, Woo H, Albuquerque F, Bozorgchami H, Dashti SR, Delgado Almandoz JE, Kelly ME, Turner R 4th, Woodward BK, Brinjikji W, Lanzino G, Lylyk P: International retrospective study of the Pipeline Embolization Device: a multicenter aneurysm treatment study. AJNR Am J Neuroradiol 2015;36:108-115.

2 Becske T, Brinjikji W, Potts MB, Kallmes DF, Shapiro M, Moran CJ, Levy EI, McDougall CG, Szikora I, Lanzino G, Woo HH, Lopes DK, Siddiqui AH, Albuquerque FC, Fiorella DJ, Saatci I, Cekirge SH, Berez AL, Cher DJ, Berentei Z, Marosfoi M, Nelson PK: Long-term clinical and angiographic outcomes following Pipeline Embolization Device treatment of complex internal carotid artery aneurysms: five-year results of the Pipeline for Uncoilable or Failed Aneurysms trial. Neurosurgery 2017;80:40-48.

3 Jiang B, Paff M, Colby GP, Coon AL, Lin LM: Cerebral aneurysm treatment: modern neurovascular techniques. Stroke Vasc Neurol 2016;1:93-100.

4 Colby GP, Lin LM, Gomez JF, Paul AR, Huang J, Tamargo RJ, Coon AL: Immediate procedural outcomes in 35 consecutive Pipeline embolization cases: a single-center, single-user experience. J Neurointerv Surg 2013;5: 237-246.

5 Colby GP, Lin LM, Caplan JM, Jiang B, Huang J, Tamargo RJ, Coon AL: Immediate procedural outcomes in 44 consecutive Pipeline Flex cases: the first North American single-center series. J Neurointerv Surg 2016;8:702-709.

6 Lin LM, Colby GP, Kim JE, Huang J, Tamargo RJ, Coon AL: Immediate and follow-up results for 44 consecutive cases of small $(<10 \mathrm{~mm})$ internal carotid artery aneurysms treated with the Pipeline embolization device. Surg Neurol Int 2013;4:114.

7 Saatci I, Yavuz K, Ozer C, Geyik S, Cekirge HS: Treatment of intracranial aneurysms using the Pipeline flowdiverter embolization device: a single-center experience with long-term follow-up results. AJNR Am J Neuroradiol 2012;33:1436-1446.

8 Bender MT, Lin LM, Colby GP, Lubelski D, Huang J, Tamargo RJ, Coon AL: P2Y12 hyporesponse (PRU > 200) is not associated with increased thromboembolic complications in anterior circulation Pipeline. J Neurointerv Surg 2017;9:978-981. 
9 Brinjikji W, Murad MH, Lanzino G, Cloft HJ, Kallmes DF: Endovascular treatment of intracranial aneurysms with flow diverters: a meta-analysis. Stroke 2013;44:442-447.

10 Patel A, Miller TR, Shivashankar R, Jindal G, Gandhi D: Early angiographic signs of acute thrombus formation following cerebral aneurysm treatment with the Pipeline embolization device. J Neurointerv Surg 2017; 9:11251130 .

11 Skukalek SL, Winkler AM, Kang J, Dion JE, Cawley CM, Webb A, Dannenbaum MJ, Schuette AJ, Asbury B, Tong FC: Effect of antiplatelet therapy and platelet function testing on hemorrhagic and thrombotic complications in patients with cerebral aneurysms treated with the Pipeline embolization device: a review and meta-analysis. J Neurointerv Surg 2016;8:58-65.

12 Park JH, Kim JE, Sheen SH, Jung CK, Kwon BJ, Kwon OK, Oh CW, Han MH, Han DH: Intraarterial abciximab for treatment of thromboembolism during coil embolization of intracranial aneurysms: outcome and fatal hemorrhagic complications. J Neurosurg 2008;108:450-457.

13 Ries T, Siemonsen S, Grzyska U, Zeumer H, Fiehler J: Abciximab is a safe rescue therapy in thromboembolic events complicating cerebral aneurysm coil embolization: single center experience in 42 cases and review of the literature. Stroke 2009;40:1750-1757.

14 Velat GJ, Burry MV, Eskioglu E, Dettorre RR, Firment CS, Mericle RA: The use of abciximab in the treatment of acute cerebral thromboembolic events during neuroendovascular procedures. Surg Neurol 2006;65:352-358, discussion 358-359.

15 Lall RR, Crobeddu E, Lanzino G, Cloft HJ, Kallmes DF: Acute branch occlusion after Pipeline embolization of intracranial aneurysms. J Clin Neurosci 2014;21:668-672.

16 Colby GP, Lin LM, Huang J, Tamargo RJ, Coon AL: Utilization of the Navien distal intracranial catheter in 78 cases of anterior circulation aneurysm treatment with the Pipeline embolization device. J Neurointerv Surg 2013; 5(suppl 3):iii16-iii21.

17 Lin LM, Colby GP, Jiang B, Uwandu C, Huang J, Tamargo RJ, Coon AL: Classification of cavernous internal carotid artery tortuosity: a predictor of procedural complexity in Pipeline embolization. J Neurointerv Surg 2015;7: 628-633.

18 Yang H, Li Y, Jiang Y: Insufficient platelet inhibition and thromboembolic complications in patients with intracranial aneurysms after stent placement. J Neurosurg 2016;125:247-253.

19 Adeeb N, Griessenauer CJ, Moore JM, Foreman PM, Shallwani H, Motiei-Langroudi R, Gupta R, Baccin CE, Alturki A, Harrigan MR, Siddiqui AH, Levy EI, Ogilvy CS, Thomas AJ: Ischemic stroke after treatment of intraprocedural thrombosis during stent-assisted coiling and flow diversion. Stroke 2017;48:1098-1100.

20 Adeeb N, Griessenauer CJ, Foreman PM, Moore JM, Shallwani H, Motiei-Langroudi R, Alturki A, Siddiqui AH, Levy EI, Harrigan MR, Ogilvy CS, Thomas AJ: Use of platelet function testing before Pipeline embolization device placement: a multicenter cohort study. Stroke 2017;48:1322-1330.

21 EPIC Investigators: Use of a monoclonal antibody directed against the platelet glycoprotein IIb/IIIa receptor in high-risk coronary angioplasty. N Engl J Med 1994;330:956-961.

22 EPIC Investigators: Platelet glycoprotein IIb/IIIa receptor blockade and low-dose heparin during percutaneous coronary revascularization. N Engl J Med 1997;336:1689-1696.

23 Fiorella D, Albuquerque FC, Han P, McDougall CG: Strategies for the management of intraprocedural thromboembolic complications with abciximab (ReoPro). Neurosurgery 2004;54:1089-1097; discussion 1097-1098.

24 Mounayer C, Piotin M, Baldi S, Spelle L, Moret J: Intraarterial administration of abciximab for thromboembolic events occurring during aneurysm coil placement. AJNR Am J Neuroradiol 2003;24:2039-2043.

25 Alexander MJ, Duckwiler GR, Gobin YP, Viñuela F: Management of intraprocedural arterial thrombus in cerebral aneurysm embolization with abciximab: technical case report. Neurosurgery 2002;50:899-901; discussion 901-902.

26 Aggour M, Pierot L, Kadziolka K, Gomis P, Graftieaux JP: Abciximab treatment modalities for thromboembolic events related to aneurysm coiling. Neurosurgery 2010;67(suppl Operative):503-508.

27 Bendok BR, Padalino DJ, Levy EI, Qureshi AI, Guterman LR, Hopkins LN: Intravenous abciximab for parent vessel thrombus during basilar apex aneurysm coil embolization: case report and literature review. Surg Neurol 2004; 62:304-311.

28 Dornbos D 3rd, Katz JS, Youssef P, Powers CJ, Nimjee SM: Glycoprotein IIb/IIIa inhibitors in prevention and rescue treatment of thromboembolic complications during endovascular embolization of intracranial aneurysms. Neurosurgery 2017, Epub ahead of print.

29 Brinjikji W, Morales-Valero SF, Murad MH, Cloft HJ, Kallmes DF: Rescue treatment of thromboembolic complications during endovascular treatment of cerebral aneurysms: a meta-analysis. AJNR Am J Neuroradiol 2015;36: 121-125.

30 Walsh RD, Barrett KM, Aguilar MI, Lanzino G, Hanel RA, Miller DA, Chong BW, Freeman WD: Intracranial hemorrhage following neuroendovascular procedures with abciximab is associated with high mortality: a multicenter series. Neurocrit Care 2011;15:85-95.

31 Ramakrishnan P, Yoo AJ, Rabinov JD, Ogilvy CS, Hirsch JA, Nogueira RG: Intra-arterial eptifibatide in the management of thromboembolism during endovascular treatment of intracranial aneurysms: case series and a review of the literature. Interv Neurol 2013;2:19-29.

32 Sedat J, Chau Y, Mondot L, Chemla R, Lonjon M, Padovani B: Is eptifibatide a safe and effective rescue therapy in thromboembolic events complicating cerebral aneurysm coil embolization? Single-center experience in 42 cases and review of the literature. Neuroradiology 2014;56:145-153. 